\title{
ARTICLE
}

\section{Estimation of neutron and gamma radiation doses inside the concrete shield wall for 10 and 15 MV medical linear accelerators}

\author{
Toshioh Fujibuchi $^{a^{*}}$, Satoshi Obara ${ }^{b}$, Masaru Nakajima $^{c}$, Nozomi Kitaura $^{c}$ and Tomoharu Sato ${ }^{c}$ \\ ${ }^{a}$ Ibaraki Prefectural University of Health Sciences, 4669-2, Ami, Ami-machi, Inashiki-gun, Ibaraki-ken, 300-0394, Japan; ${ }^{b}$ National \\ Institute of Radiological Sciences, 4-9-1, Anagawa, Inage-ku, Chiba,Chiba-ken, 263-8555, Japan; 'Center Institute Hospital, \\ Japanese Foundation for Cancer Research, 3-8-31,Ariake, Koto-ku, Tokyo, 135-8550, Japan
}

\begin{abstract}
High-energy electron linear accelerators (linacs) are used in radiotherapy for tumor treatment. By operating at electron energies above the thresholds of the $(\gamma, n)$ photonuclear reaction, undesirable photoneutrons and radioisotopes are generated by high-energy x-rays. These photonuclear reactions occur on the linac accelerator head components, which are made from various materials. In these reactions, one can assume that secondary neutrons are dispersed all over the treatment rooms. The present work observed ambient dose equivalent of photon and neutron, and number of etch pits of wide-energy range Neupit produced by thermal and fast neutrons inside a model of iron shielding wall. The neutron dose around the surface of the shielding wall was detected less than three orders of magnitude of photons at $10 \mathrm{MV}$ linac. The total neutron dose in a $15 \mathrm{MV}$ linac was about one order higher than that in the $10 \mathrm{MV}$ linac. In addition, the $15 \mathrm{MV}$ linac incident photons clearly generated secondary neutrons inside the iron shielding wall.
\end{abstract}

Keywords: medical linear accelerator; iron shield wall; photonuclear reaction; neutron

\section{Introduction}

External beam radiotherapy is the most common form of radiotherapy for tumor treatment. High-energy electron linear accelerators (linacs) are used in external beam radiotherapy. Since the threshold for photoneutron production on lead is about $8 \mathrm{MeV}$, contamination due to neutrons is not a problem for therapy beams below 10 MV. At this energy, the production of neutrons is still quite low, but should be taken into account. By $15 \mathrm{MV}$, neutron production increases by a factor of ten for 10 $\mathrm{MV}$, and by $18 \mathrm{MV}$ a further factor of two for $15 \mathrm{MV}$ [1]. Few measurements and calculations for the neutron dose rate distribution around linac shielding walls have been conducted previously [2-4]. Considering the large discrepancy reported between the radiation dose results of calculations and measurements [5], the generation of photoneutrons and several radioisotopes inside the shielding wall should be taken into account when the therapeutic beam energy is higher than the nuclear reaction thresholds [6]. By further increase of the incident photon energy, photoneutrons are produced in the structure materials such as iron of shielding wall.

As far as radiation dose from the target is concerned, the dose of photoneutrons is smaller than the dose of photon, and can be ignored. However, shielding calculations considering only photons from the target

*Corresponding author. Email: fujibuch@hs.med.kyushu-u.ac.jp have been performed and found out that the measured dose was greater than the calculated ones by about two orders of magnitude [6]. The literature evaluated behavior of radiation inside of wall by Monte-Carlo calculation and measured leakage dose using ion chamber and rem meter. Oishi et al. measured and dose rate distribution in the laminated shielding assembly irradiated by 18, 25 and $34 \mathrm{MeV}$ photons [7]. But they did not measured photon and neutron dose inside shielding wall from 10 and $15 \mathrm{MV}$ linac.

The purpose of this study was to increase the understanding of neutron behavior inside the shielding wall of a radiotherapy treatment room. In this study, we measured photon and neutron exposure dose inside a model of iron shielding wall.

\section{Material and methods}

\subsection{Experiments in 10 and 15 MV linacs}

Photoneutrons are mainly induced in the high- $Z$ materials on the linac head such as the target, the flattening filter, the jaws, the multi leaf collimators, and the head shielding [8]. Experiments were performed for two types of linacs to evaluate the energy dependence of the secondary neutrons generated inside the model of shielding wall. The accelerating electron energy of the linac at Ibaraki Prefectural University of Health Sciences was $10 \mathrm{MV}$ (EXL-15SP: Mitsubishi Electric 


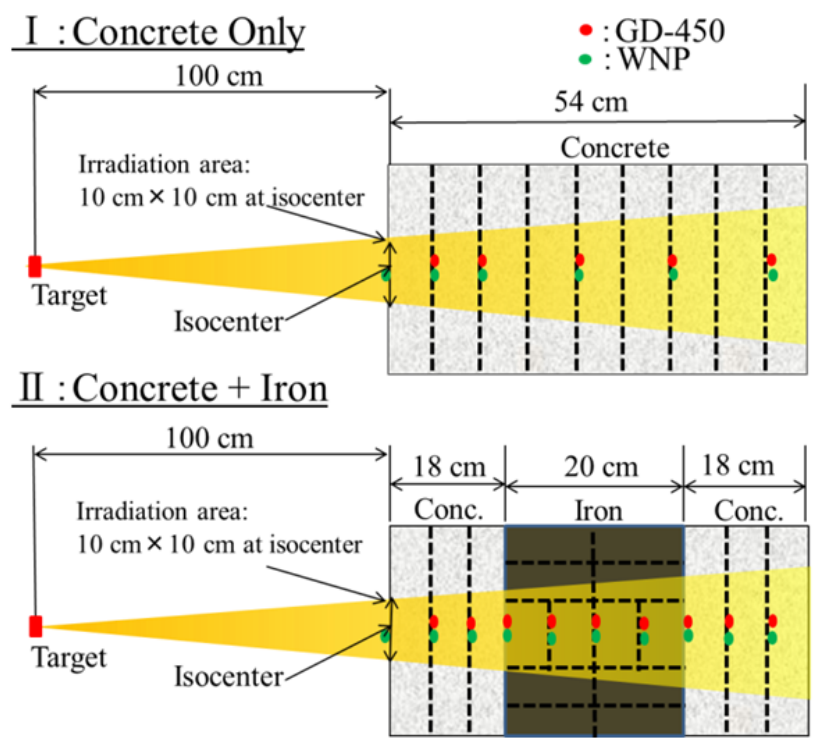

Figure 1. Experimental shield array of the concrete and iron shielding (cross-sectional plane view).

Co.) with copper target and tungsten collimators, and that of the linac at the Japanese Foundation for Cancer Research, Center Institute Hospital of Radiation Oncology, Tokyo was 15 MV (CLINACix: Varian Medical systems) with tungsten and copper target, and tungsten collimators.

Shielding for neutrons requires hydrogen-containing material and that for photons requires high- $Z$ material. Experiments were performed for a pair of models to allow a comparison between concrete-block shielding and shielding composed of iron sandwiched between concrete blocks. The experimental arrangement is shown in Figure 1. The layout II is configured to simulate a typical concrete shielding wall with built-in iron gamma-ray shielding. The photon beam and other scattered or photonuclear production particles were irradiated on the surface of the shielding wall perpendicularly at its center. The irradiated area was 10 $\mathrm{cm} \times 10 \mathrm{~cm}$, and the surface cross section of the shielding was $30 \mathrm{~cm} \times 30 \mathrm{~cm}$. The total thickness of the concrete-iron shielding wall was $56 \mathrm{~cm}$, where two 18 -cm-thick concrete layers sandwiched a 20 -cm-thick iron layer. Each concrete layer consisted of three slabs cut to $6 \mathrm{~cm}$ thickness with an area of $30 \mathrm{~cm} \times 30 \mathrm{~cm}$ and density is $2.1 \mathrm{~g} / \mathrm{cm}^{3}$. The concrete is a composition of four components blended in a certain proportion: cement, crushed stones, sand, and water. The main weight compositions of concrete are calcium (41.6\%), silicon (11.0\%), aluminum $(2.3 \%)$, iron $(2.2 \%)$, sulfur $(1.4 \%)$, potassium $(0.56 \%)$, phosphorus $(0.6 \%)$ and sodium $(0.08 \%)[9]$. The weight compositions of hydrogen, the carbon, and oxygen are not measured. The array of iron blocks $\left(5 \mathrm{~cm} \times 10 \mathrm{~cm} \times 20 \mathrm{~cm}, 7.9 \mathrm{~g} / \mathrm{cm}^{3}\right)$ was $20 \mathrm{~cm}$ in thickness.

\subsection{Measurements of photon and neutron dose rate distributions inside the shielding wall}

In this study, we used Glass Badge using silver activated phosphate glass dosimeter (GD-450: Chiyoda Technol Co.) for photon dose measurement, and wide-energy range Neupit (WNP: Chiyoda Technol Co.) that is allyl diglycol carbonate plastic solid state nuclear track detector for neutron dose measurement. They were specially developed for large scale personal monitoring. GD-450 has two plastic filters and three metal filters (aluminum, copper and tin), and this composition enables to measure $10 \mathrm{keV}$ to $10 \mathrm{MeV}$ photon and 300 $\mathrm{keV}$ to $3 \mathrm{MeV}$ beta radiation [10]. GD-450 size is 45 $\mathrm{mm} \times 13 \mathrm{~mm} \times 5 \mathrm{~mm}$ and $5 \mathrm{~g}$ weight. WNP has two kind of charged particle radiator - boron nitride (BN) and polyethylene - to cover wide energy range of neutron measurement such as $0.025 \mathrm{eV}$ to $15 \mathrm{MeV}$ continuously[10, 11]. WNP size is $27 \mathrm{~mm} \times 9 \mathrm{~mm} \times 2$ $\mathrm{mm}$ and $0.5 \mathrm{~g}$ weight. GD-450 and WNP were placed between the concrete slabs and in the iron array. The measurement points were shown in Figure 1. Use of the WNP that is commonly employed to detect wide energy ranges of neutrons and various particles is suitable for simple and easy measurements. The analysis of measured GD-450 and WNP were executed by Chiyoda technol corporation.

\section{Results and discussion}

\subsection{Photon and neutron ambient dose equivalent inside a model of shielding wall}

The experimental values for both photon and neutron dose equivalents inside a model of shielding wall were normalized to a monitor unit (MU). A MU is the amount of charge recorded in the ionization chamber mounted in the head of linac. Linac is calibrated to give a particular absorbed dose under particular conditions. $100 \mathrm{MU}$ gives an absorbed dose of $1 \mathrm{~Gy}$ at isocenter, for a $10 \mathrm{~cm}$ $\times 10 \mathrm{~cm}$ field. The isocenter is the point in space where radiation beams intersect when the gantry is rotated during beam-on.

GD-450 and WNP were calibrated on a condition of free air, and they gave the ambient dose equivalents in this measurement. We observed that the attenuation of photon and neutron doses depended on the observation distance from the surface of the shielding wall.

Figure 2 shows the photon ambient dose equivalent inside the shielding walls of two configurations and two energies. The photon doses of 10 and $15 \mathrm{MV}$ were in agreement. The photon attenuation profiles of the dose equivalent inside the concrete-only shielding wall decreased exponentially.

Figure 3 shows the neutron ambient dose equivalent inside the shielding walls. The neutron dose equivalent increased inside the iron shielding wall for both electron energies. The neutron dose equivalent was increased by the inserted iron slab about ten times in $15 \mathrm{MV}$ and 3 times in $10 \mathrm{MV}$. The reason the neutron dose increases includes photonuclear reaction of iron. We did not measure energy spectrum of incident photons; however, the dose increase suggested that even the $10 \mathrm{MV}$ linac emitted photons of energies higher than the threshold 


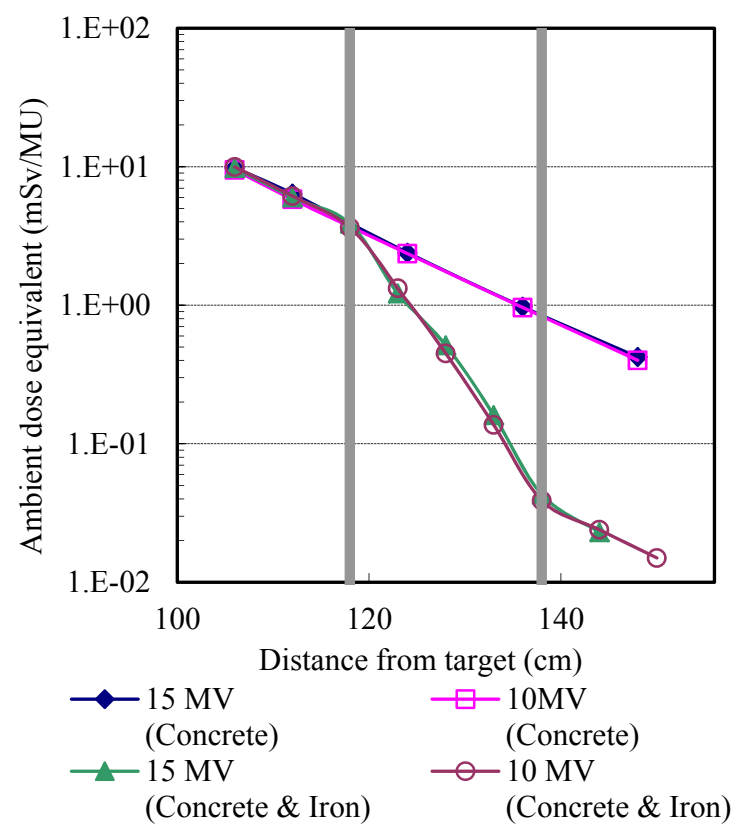

$\rightarrow$ Boundary of iron and concrete

Figure 2. Photon ambient dose equivalent inside a model of shielding wall.

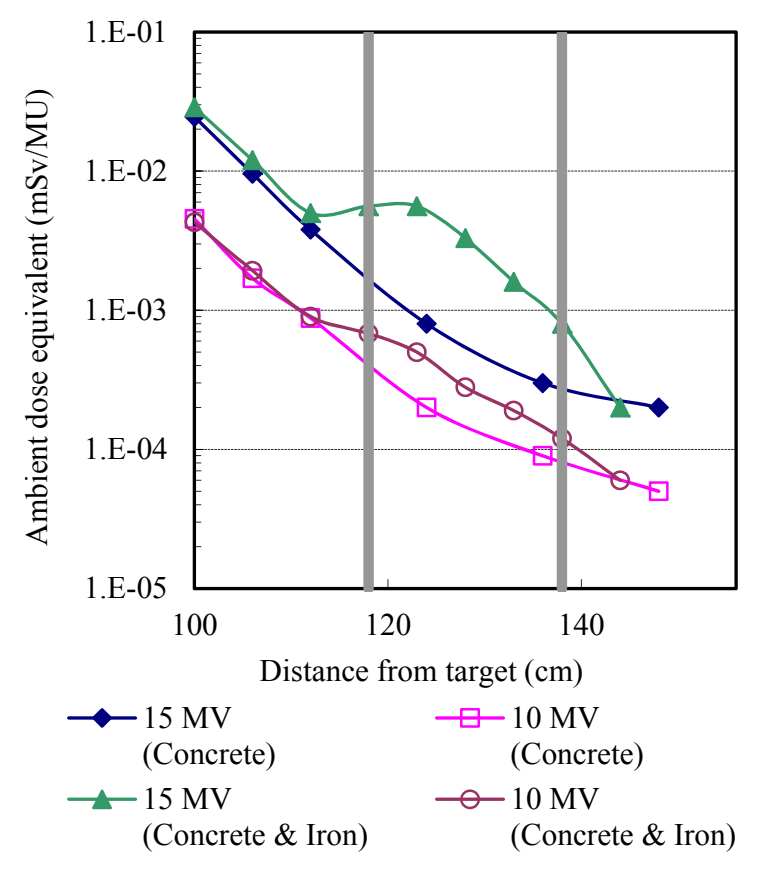

Boundary of iron and concrete

Figure 3. Neutron ambient dose equivalent inside a model of shielding wall.

energy of iron $(\gamma, \mathrm{n})$ reaction $\left(7.6 \mathrm{MeV}\right.$ for ${ }^{57} \mathrm{Fe}$ [abundance:2.1\%], $10 \mathrm{MeV}$ for ${ }^{58} \mathrm{Fe}[0.28 \%], 11.2 \mathrm{MeV}$ for ${ }^{56} \mathrm{Fe}[91.6 \%], 13.4 \mathrm{MeV}$ for $\left.{ }^{54} \mathrm{Fe}[5.6 \%]\right)$. Moreover, attenuation of the neutron in concrete is larger than iron [7]. It is thought that the difference of attenuation with the medium also influences.

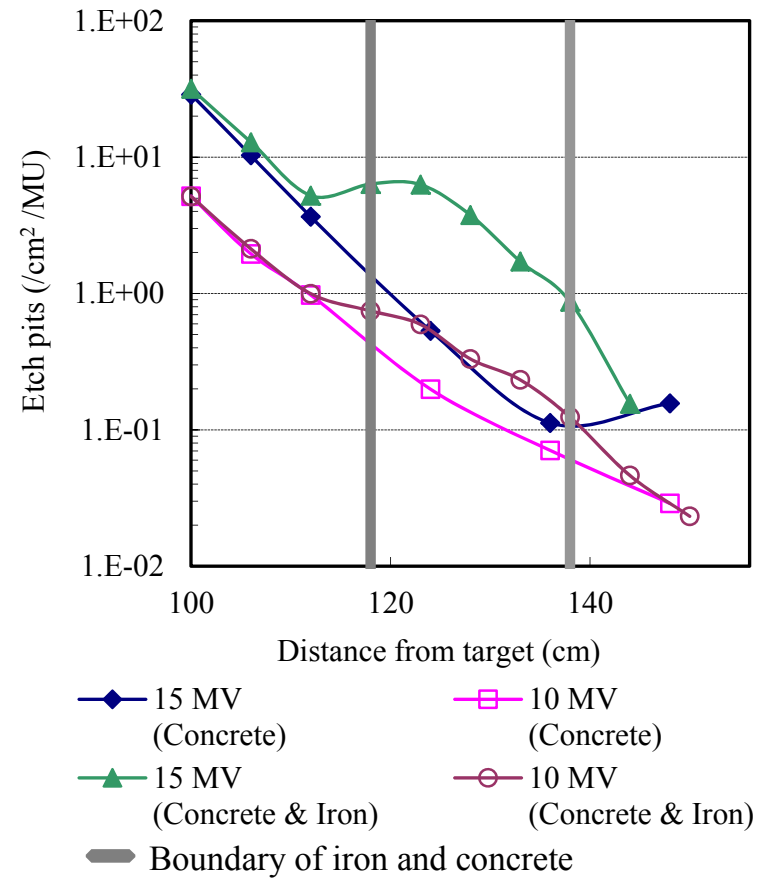

Figure 4. Number of etch pits of WNP produced by fast neutrons inside a model of shielding wall.

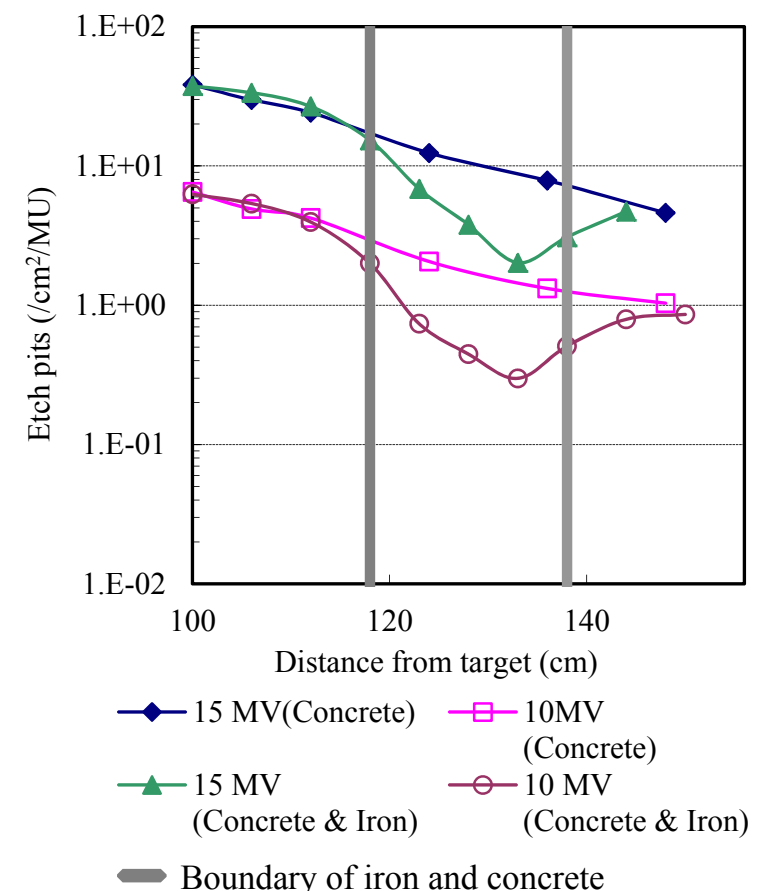

Figure 5. Number of etch pits of WNP produced by thermal neutrons inside a model of shielding wall.

In this study, the neutron dose equivalent rate decreased to about $1 / 100$ in the front and the rear shielding block at 10 and $15 \mathrm{MV} \mathrm{X}$ ray. The block was composed of the concrete of $36 \mathrm{~cm}$ and the iron of 20 $\mathrm{cm}$. According to Oishi et al. thesis, the neutron dose equivalent rate decreased to about $1 / 100$ in the front and 
the rear concrete wall at $18 \mathrm{MV} \mathrm{X}$ ray [6]. The wall was composed of the concrete of $54 \mathrm{~cm}$ and the iron of 66 $\mathrm{cm}$. The decrease in the dose rate was almost the same though our case was about a half in the thickness of the shielding. Oishi et al. calculates the attenuation of the neutron and photon dose rate in the shielding wall to the incidence of the photon of $18 \mathrm{MV}$. It agreed in the point that the rise of the neutron dose in front side of iron, and the sudden attenuation in a rear concrete block. The neutron dose equivalent rate in the front and the rear shielding is thought that the difference of the cross section of an photonuclear reaction by the energy of entering $\mathrm{X}$ rays influences.

The neutron dose measured in present work was $2.0 \times 10^{-5} \mathrm{~Sv} / \mathrm{MU}$ in the $15 \mathrm{MV}$ linac, and $0.4 \times 10^{-5}$ $\mathrm{Sv} / \mathrm{MU}$ in the $10 \mathrm{MV}$ linac at the position of the isocenter. Radiation Shielding Information Center Code Package [12] shows $3.0 \times 10^{-5} \mathrm{~Sv} / \mathrm{MU}$ in the $15 \mathrm{MV}$ and $0.3 \times 10^{-5} \mathrm{~Sv} / \mathrm{MU}$ in the $10 \mathrm{MV}$, it agreed almost. This kernel integration code system for multi- group gamma-ray scattering calculations using the GP buildup factor was contributed by Japan Atomic Energy Research Institute which based on CCC-75/G3-6ED, contributed by Los Alamos National Laboratory. The Q values ranged from 0.06 for a $10 \mathrm{MV}$ beam to 0.76 $\left(\times 10^{12}\right)$ neutrons per photon Gy for a $15 \mathrm{MV}$ beam of Varian. They measured number of fast neutron fluence using the gold-foil activation technique [13].

\subsection{Fast and thermal neutron behaviors inside a model of shielding wall}

Figures 4 shows the number of etch pits of WNP produced by fast neutrons inside a model of shielding wall. The etch pits are decreased exponentially at the model of concrete-only shielding wall except deepest position of $15 \mathrm{MV}$.

The reason of this phenomenon is thought that the photoneutrons generated at the target and the linac head entered through the back surface of the shield due to the room scattering. It is thought that the scattering radiation can be prevented from contributing by arranging the fast neutron moderator with polyethylene and the thermal neutron absorption material with boron behind the concrete brocks.

The attenuation of the fast neutron was different in 10 $\mathrm{MV}$ and $15 \mathrm{MV}$. The reason is thought that the neutron energy by the model is different. According to Howell et. al [14], the neutron average energies of $15 \mathrm{MV}$ linacs are $0.27 \mathrm{MeV}$ at Varian, $0.20 \mathrm{MeV}$ at Siemens, and 0.23 $\mathrm{MeV}$ at Elekta. Energy were almost the same though the manufactures were different. According to Kim et. al [15], they found the neutron average energy at isocenter for a $0 \times 0 \mathrm{~cm}^{2}$ closed field is $0.38 \mathrm{MeV}$ for $10 \mathrm{MV}$ and is $0.45 \mathrm{MeV}$ for $15 \mathrm{MV}$.

The results for the concrete-iron shielding wall showed the energy dependence of the incident photons. On the basis of this information, we conclude that a photonuclear reaction occurred inside the iron shielding wall, in which fast neutrons were generated by incident photons mainly from the target.

Figure 5 shows the number of etch pits of WNP produced by the thermal neutrons are decreased exponentially in the concrete-only shield. The decrease of thermal neutrons in iron slabs was remarkable, however, they increased again in back concrete part. The reason can be thought that the fast neutrons permeated the rear concrete slab were moderated to thermal neutrons, and the thermal neutrons permeated backward the iron slab again. And thermal neutron scatters in the room and incident to the rear slab, because the space exists during the blocks where the CR-39s were arranged in this experiment system.

\section{Conclusion}

In the $10 \mathrm{MV}$ linac, the dose contribution of secondary neutrons to the total dose was negligibly small. The number of WNP etch pits produced by fast neutrons increased in and near the iron slab with the photoneutron production, however, that produced by thermal neutrons decreased.

\section{References}

[1] M.M. Elsalim, Characterization of the neutron environment around Varian medical electron accelerators, M.S. thesis, San Jose State University, (1994).

[2] Y. Uwamino, T. Nakamura, T. Ohkubo and A. Hara, Measurement and calculation of neutron leakage from a medical electron accelerator, Medical Physics 13 (1986), pp.374-384.

[3] T. Nakamura and Y. Uwamino, Shielding design and detection of neutrons from medical and industrial electron accelerators--simple method of design calculation for neutron shielding, Radioisotopes 35 (1986), pp.51-56.

[4] P. H. McGinley, Shielding Techniques for Radiation Oncology Facilities, Medical Physics Pub Corp, 2 edition, (2002).

[5] K. Masumoto, A. Toyoda, K. Eda and T. Ishihara, Measurement of the Spatial Distribution of Neutrons in an Accelerator Room by the Combination of Activation Detectors and an Imaging Plate. Radiation Safety Management 1 (2002), pp.12-16.

[6] K. Oishi, K. Kosako, Y.Kobayashi, I. Sonoki and T. Nakamura, Consideration to Shielding Design Calculation of Radiation Oncology Electron Linac Facility whose Energy is Greater than $10 \mathrm{MeV}$. Radioisotopes 57 (2008), pp.277-286.

[7] K. Oishi, K. Kosako, T. Nakamura, Y. Kobayashi, I. Sonoki, K. Sato, T. Kamiyama and Y. Kiyanagi: Measurement and Analysis of Dose Rate Distribution in the Laminated Shielding Assembly Irradiated by High Energy Photons, J. Nucl. Sci. and Technol., Supplement 5 (2008), pp.32-35.

[8] H. R. Vega-Carrillo, S. A. Martı'nez-Ovalle, A. M. Lallena, G. A. Mercado and J. L. Benites-Rengifo, 
Neutron and photon spectrain LINACs, Applied Radiation and Isotopes, (2012).

[9] J. C. Evans, E. L. Lepel, R. W. Sanders, C. L. Wilkerson, W. Silker, C. W. Thomas, K. H. Abel and D. R Robertson, Long-lived activation products in reactor materials, NUREG/CR-3474, (1984).

[10] N. Juto, The large scale personal monitoring service using the latest personal monitor glass badge, paper for proceedings of AOCRP-1, (2002).

[11] H. Ohguchi, and T. Nakamura, Development of Wide-Energy Range Personal Neutron Dosimeter Using CR-39 Track Detector, Appl. Radiat. Isot. 46 (1995), pp.509-510.

[12] Radiation Shielding Information Center Code Package, CCC-494/G33-GP, (1986).
[13] D.S. Followill, M.S. Stovall and S.F. Kry, Neutron source strength measurements for Varian, Siemens, Elekta, and General Electric linear accelerators, Journal of Applied Clinical Medical Physics 4(3) (2003), pp.189-194.

[14] R. M. Howell, S. F. Kry, E. Burgett, N. E. Hertel and D. S. Followill, Secondary neutron spectra from modern Varian, Siemens, and Elekta linacs with multileaf collimators, Medical Physics 36 (2009), pp.4027-4038.

[15] H. S. Kim, Y.H. Park, B. C. Koo, J. W. Kwon, J. S. Lee and H. S. Choi, Evaluation of the photoneutron field produced in a medical linear accelerator, Radiat Prot Dosimetry 123(3), (2007), pp.323-328. 